\title{
Age Group Recognition using Human Facial Images
}

\author{
Shailesh S. Kulkarni \\ Dept. of Electronics and Telecommunication \\ Government College of Engineering, \\ Aurangabad, Maharashtra, India
}

\begin{abstract}
Recognizing human age group automatically through facial image analysis has many applications, such as human computer interaction and multimedia communication. The aging process involves many factors such as the person's gene, health, living style, living location and weather conditions. This paper presents an automatic human age group Recognition system based on human facial images. Features are extracted using two approaches namely Principal Component Analysis (PCA) coefficients and Discrete Cosine Transform (DCT) coefficients and the classification is done using Euclidian Distance classifier. The results shows DCT based approach performs better as compared to results using PCA.
\end{abstract}

\section{Keywords}

HCI, PCA, DCT, Euclidean Distance, Age classification, Facial Images, MPCA

\section{INTRODUCTION}

Human can convey many kinds of useful information from face images such as identity, gender, approximate age and expression. From last decades researchers have work on automatic age detection topic in the Human Computer Interaction (HCI) field. Face images plays a vital role in face to face communication between human beings. Even if the aging pattern is different for each person, individuals belonging to the same age group share some facial shape and texture similarities.

In the earlier work of age estimation researchers mainly used two publically available databases namely MORPH and FGNET [5-6]. The MORPH Database consists of two sections namely section I and II. It consists of images in age range from 15 to 68. The FG-NET (Face and Gesture Recognition Research Network) aging database consists of 1002 images of 82 subjects (6 to 18 images per subject) in the age range 0 to 69 years. Sample images from the FGNET dataset are shown in figure 1 .

Various types of Feature extraction techniques are studied which includes Active Appearance Model (AAM), Principal Component Analysis (PCA), Discrete Wavelet Transform (DWT), discrete cosine Transform (DCT), Local Binary Pattern (LBP), etc. The researchers explored many classification methods for classifying extracted features into age groups, such as k-nearest neighbors $(\mathrm{KNN})$, Support Vector Machine (SVM), Artificial Neural Network (ANN), Euclidian Distance Classifier etc. [10, 14, 16-19].



Fig. 1. Sample images of same individual at different ages in FGNET database with corresponding age value.

Age group recognition system can be used for many applications such as System access control where young kids can be denied to access adult websites, sell of cigarettes and alcohol from vending machine to underage people can be declined, age of people who spend more time to see particular advertisement can be find out by Ad-agency, the videos and photographs of users can be reclaimed by specifying particular age group [9],[11]. Apart from this it has application in the area of Forensic Art and cosmetology [8] and for interactive games where an intelligent toy can automatically provide different games based on the player's age range [13]

Rest of the paper is arranged as follows. Section 2 shows literature survey. Section 3 illustrates architecture of Age group Recognition system. Section 4 consists of Experimental work and results and finally, section 5 concludes the paper.

\section{LITERATURE SURVEY}

In the past few years several approaches have been proposed for studying facial age group recognition. Some important contributions from the existing literature are briefed in this section. The first work on the age classification problem is done by Kwon and Lobo. They classified gray scale facial images into three age groups: babies, young adults and senior adults. First, primary features (such as eyes, noses, mouth, etc.) from a facial image were located by applying deformable templates and snakes, and judged if it is an infant by the ratios of the distances between primary features [7].Vahid Karimi et al. experimented on an automatic system in which major features from facial images are extracted and then by calculating major ratios, the age and gender are estimated [2].V.Tamil Selvi et al. have proposed the age estimation system based on MPCA method. They used Gabor feature extraction method and Artificial Neural Networks. The advantage of this method is the timing complexities are reduced [1].Guo et al. introduced the age manifold learning technique for extracting face aging features and designs a locally adjusted robust regressor for learning and prediction of 


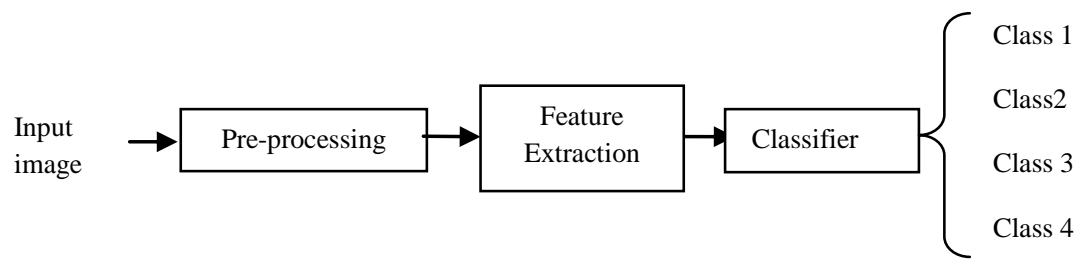

Fig 2: Architecture of Age group Recognition System

human ages. They used an internal age database UIUC-IFP-Y and publicly available FG-NET database. Age estimation experiments are done on female and male images separately [4]. Xin Geng et al. have stated that the recognition of most facial variations, such as identity, expression, and gender, has been broadly studied. They presented an automatic age estimation technique called AGES (AGing pattern Subspace) [3].

\section{THE ARCHITECTURE OF AGE GROUP RECOGNITION SYSTEM}

The architecture of age group Recognition system consists of four components: input image, pre-processing, feature extraction and classification as shown in figure 2.

\subsection{Preprocessing}

Before using the images for feature extraction it needs to be normalized it is because input images are affected by the type of camera, Illumination conditions and background information. The database which is used in this work consists of images of varying size, so all the images are resized to $420 \times 360$. The color images are converted into Gray Scale images for easier processing. This is the initial step and hence to be done at the beginning, further transformations are done using these gray scaled images

\subsection{Feature Extraction}

Information extracted from an image is used to represent the image in Recognition problems such as Age Group Recognition. This information also referred as features which may take each individual image pixels independently or features extracted in such a way to capture patterns represented in neighboring pixels. In this paper, we have considered two feature extraction techniques namely Principal component Analysis (PCA) coefficients and Discrete Cosine Transform (DCT) coefficients.

\subsubsection{Principal Component Analysis (PCA)}

Principal Component Analysis (PCA) is an orthogonal transform that converts data to new coordinate system in such a way that the first coordinate consists of data with largest variance, the second coordinate consists of data with second largest variance and so on[12]. PCA can be used for prediction, redundancy removal, feature extraction, compression etc. following sequence of steps are to be performed while using PCA for recognition task such as age group recognition

Let $X$ is the image vector of size $m \times n$. If the training set contains such $\mathrm{N}$ images then mean image for such training set can be obtained as

$$
\overline{\mathrm{X}}=\frac{1}{\mathrm{~N}} \sum_{\mathrm{i}=1}^{\mathrm{N}} \mathrm{X}_{\mathrm{i}}
$$

Then mean subtracted image is generated to minimize the noise using following equation.

$$
\mathrm{X}=X_{i}-\overline{\mathrm{X}}
$$

To collect the distributed features from mean subtracted image covariance matrix is to be calculated using below mentioned equation

$$
\mathrm{C}=\frac{1}{N} \sum_{i=1}^{N} X^{T} \cdot \mathrm{X}
$$

Since the covariance matrix and Eigen values are related by following identity:

$$
\mathrm{CV}=\lambda \mathrm{V}
$$

Where $\mathrm{V}$ is the set of eigenvectors corresponding to its eigen values .once eigenvectors and eigen values are obtained, they are descended since the Eigen vectors corresponding to highest eigen values are most important. Finally we obtained feature vector of training set as:

$$
W_{i}=V_{i}^{\mathrm{T}} \mathrm{X}
$$

In the testing phase each test image should be mean centered, than projected into the same Eigen space as defined during the training phase. This projected image is than compared with projected training image in Eigen space. Images are compared with similarity measures. The training image that is closest to the test image will be matched and used to identify.

\subsubsection{Discrete Cosine Transform (DCT)}

DCT is widely used as a feature extraction and compression method in various image processing applications due to its properties such as de-correlation, separability and orthogonality.DCT has excellent energy compaction capability. This technique takes the transformation of an image resulting into three frequency components namely low, middle and high. DCT packs energy in the low frequency regions. Therefore some of the high frequency content can be discarded without significant quality degradation. If $f(\mathrm{x}, \mathrm{y})$ is the intensity of the pixel at coordinates $(\mathrm{x}, \mathrm{y}), \mathrm{u}$ varies from 0 to $\mathrm{M}-1$ and $\mathrm{v}$ varies from 0 to $\mathrm{N}-1$.Mathematically, the 2DDCT of an image is given by: 
$F(u, v)=\alpha(u) \alpha(v) \sum_{x=0}^{N-1} \sum_{y=0}^{M-1} \cos \left[\frac{\pi u}{2 N}(2 x+1)\right] \cos \left[\frac{\pi u}{2 M} \cos (2 y+1)\right] f(x, y)$

Where,

$\alpha(u) \alpha(v)=\sqrt{\frac{1}{N}}$ for $u, v \neq 0$

$\alpha(u) \alpha(v)=\sqrt{\frac{2}{N}}$ for $u, v \equiv 0$

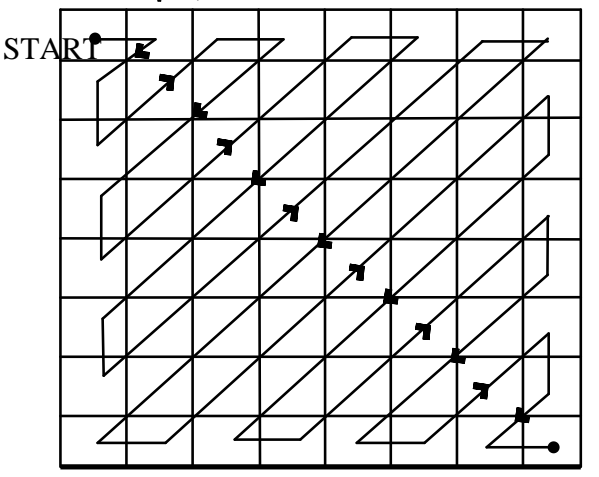

END

Fig 3: zig-zag scanning order used for selection of DCT coefficient

After applying DCT in the face images we can see that most of the energy of the coefficients is concentrated in the few low-frequency components of the DCT or near the origin. Due to this reason selection of the important coefficients for the construction of feature vectors become easy. In most of the DCT based applications the features are extracted using zigzag scanning as shown in Fig.3.

\subsubsection{Classifier}

The method presented in this paper uses Euclidian Distance classifier for classification of an image into different age groups. For improvement of accuracy more complex classifier could be used, but this may increase the complexity of system [14][16]. A novel competitive nearness approach is implemented using the average class distance. The average for each of the four training class is calculated. Then for any test input image, the distance to these four class's average sets were computed. The class which has the least distance was considered to be the age result. And the age range label was assigned based on the label of the aging group.

Let us consider $\mathrm{x}_{\mathrm{r}}$ is training feature vector which is stored into database and $y_{t}$ is the test feature vector of the image being tested against the system. Euclidean distance between pair of vectors $E_{d}$ is given by following mathematical equation as:

$E_{d}=\sqrt[2]{\sum\left(x_{r}-y_{t}\right)^{2}}$

Minimum Euclidean distance decides the Age Group of the test sample.

\section{EXPERIMENTAL WORK AND RESULT}

This section describes experimental setup and discusses results obtained with both the approaches.

\subsection{Experimental Setup}

In this work, instead of trying to estimate the age of a person, age group is considered for recognition. following four age group categories are considered for recognition.
1. Baby and child: $0-10$ years of age.

2. Young: $11-18$ years of age.

3. Adult: $19-39$ years of age.

4. Middle and Old age: $40-60$ years of age

The images used for training are from FGNET [5] Database. The images in this Database are not equally distributed over age ranges. As the images were retrieved from real-life albums of different subjects, aspects such as illumination, head pose, facial expressions etc. are uncontrolled in this dataset. In fact, this database is the only publicly available resource that provides images of individuals in the range 0-18 years. The distribution of images in different age groups is as shown in Table 1.

Table 1.Database specifications and details

\begin{tabular}{lr|c}
\hline \multicolumn{2}{l|}{ Age Classes } & Number of samples \\
\hline Baby and Child (0-10) & 411 \\
Young (11-18) $\quad(19-39)$ & 276 \\
Adult $\quad 253$ \\
Middle and old Age(40-69)
\end{tabular}

Experiments are carried out using two approaches to tabulate the recognition performance. The two approaches that use principal component analysis coefficients and discrete cosine transform coefficients .in each case training set consists of 50 images from each group totaling to 200 images. Each detected face is scaled to $320 \times 240$.

To check the performance of the system the test set consists of total of 60 images, 15 images from each group. Recognition rate is calculated in either case.

Recognition Rate $=\frac{\text { correctly recognized test images }}{\text { Total number of test images }}$

When using DCT coefficients as a features the size of feature vector is based on the selection of DCT patch size whereas in PCA based approach originally feature vector size is 76800 which is reduced by using PCA for dimensionality reduction resulting from the image of size $320 \times 240$.

When using DCT coefficients as a features the size of feature vector is based on the selection of DCT patch size whereas in PCA based approach originally feature vector size is 76800 which is reduced by using PCA for dimensionality reduction resulting from the image of size $320 \times 240$.

\subsection{Results and Discussion}

Results obtained by using PCA coefficients as features are shown in table 2.Class 4 , show larger value of recognition rate i.e. $66.67 \%$ whereas for class 1 lowest recognition rate of $46.66 \%$ is achieved.

Average recognition rate using PCA based approach is $56.66 \%$. This table shows the lowest recognition rate is obtained for child class this is due to the confusion of system between child class and young class. Almost all the misclassified images from child class are identified as young class. 
Table 2.PCA Recognition Rate

\begin{tabular}{|c|c|c|c|}
\hline \multirow[b]{2}{*}{$\begin{array}{c}\text { Age } \\
\text { Group }\end{array}$} & \multicolumn{2}{|c|}{ Recognition Result } & \multirow{2}{*}{$\begin{array}{c}\text { Average Correct } \\
\text { Recognition Rate } \\
(\%)\end{array}$} \\
\hline & $\begin{array}{c}\text { Correct } \\
\text { Rate }(\%)\end{array}$ & $\begin{array}{l}\text { Incorrect } \\
\text { Rate (\%) }\end{array}$ & \\
\hline Child & 46.66 & 53.34 & \multirow{4}{*}{56.66} \\
\hline Young & 60.00 & 40.00 & \\
\hline Adult & 53.33 & 46.67 & \\
\hline $\begin{array}{l}\text { Middle \& } \\
\text { Old Age }\end{array}$ & 66.67 & 33.33 & \\
\hline
\end{tabular}

Table 3 shows results obtained by using DCT coefficients as features with DCT patch size of 20 which results feature vector of size 400 DCT coefficients.

Table 3. DCT Recognition Rate

\begin{tabular}{|c|c|c|c|}
\hline \multirow[b]{2}{*}{$\begin{array}{c}\text { Age } \\
\text { Group }\end{array}$} & \multicolumn{2}{|c|}{ Recognition Result } & \multirow{2}{*}{$\begin{array}{l}\text { Average Correct } \\
\text { Recognition Rate } \\
(\%)\end{array}$} \\
\hline & $\begin{array}{c}\text { Correct } \\
\text { Rate }(\%)\end{array}$ & $\begin{array}{l}\text { Incorrect } \\
\text { Rate }(\%)\end{array}$ & \\
\hline Child & 80.00 & 20.00 & \multirow{4}{*}{76.75} \\
\hline Young & 73.33 & 26.67 & \\
\hline Adult & 87.00 & 13.00 & \\
\hline $\begin{array}{c}\text { Middle \& } \\
\text { Old Age }\end{array}$ & 66.67 & 33.33 & \\
\hline
\end{tabular}

As shown in above table the highest recognition rate of $87 \%$ is achieved for class 3 whereas for class 4 the lowest recognition rate of $66.67 \%$ is achieved. Average recognition rate using DCT based approach is $76.75 \%$.Compared to the results of the PCA based approach the results are quite better for all the classes.

\section{CONCLUSION}

This paper gives the information about technique which classifies an image into age groups using Principal Component Analysis (PCA) and Discrete Cosine Transform (DCT) coefficients. For variable facial expressions, by using FGNET database the accuracy $76.66 \%$ is achieved. The Final result shows that the recognition rate in DCT is almost $15 \%$ more than that of PCA. The recommended DCT patch size in the range 15 to 25 but it may adversely affect recognition time. The recognition accuracy can be improved by considering larger training set and better classifier. Also work can be done to reduce average recognition time.

\section{REFERENCES}

[1] Tamil, V.S.,Vani, K. 2011.Age Estimation System using MPCA. In Proceedings of International Conference on Recent Trends in Information Technology, ICRTIT 2011, pp. 1055-1060.

[2] Vahid, K., Ashkan,T. 2012.Age and Gender Estimation by Using Hybrid Facial Features. In IEEE Conf on Telecommunications forum, pp. 1725-1728.

[3] Xin, G., Kate, S.M. 2007 .Automatic Age Estimation Based on Facial Aging Patterns. In IEEE Transactions on pattern analysis and machine intelligence, vol. 29, pp. 2234-2240.
[4] FG-Net aging database, http://sting.cycollege.ac.cy/alanitis/fgnetaging

[5] Ricanek, K., Tesafaye, T. 2006. MORPH A longitudinal image database of normal adult age-Progression. In Proceedings of International Conference on Automatic Face and Gesture Recognition, pp. 341-345.

[6] Kwon, Y., Lobo, N. 1999. Age classification from facial images. Computer Vision and Image Understanding, vol. 74 , no. 1, pp. 1-21.

[7] Yun, F., Guodon, G. 2010 . Age Synthesis and Estimation via Faces: A Survey, IEEE transactions on pattern analysis and machine intelligence, vol. 32, pp. 1955-1976.

[8] Fu , Y., Huang, T. S. 2007. Estimating human age by manifold analysis of face pictures and regression on aging features. In IEEE Conf. ICME’07, 2007, pp. 13831386.

[9] Lanitis, A., Draganova, C. 2004. Comparing Different Classifiers for Automatic Age Estimation. In IEEE transactions on systems, man, and cybernetics, vol. 34, pp. 621-628.

[10] Guo, G., Dyer, C. 2008. Locally adjusted robust regression for human age estimation. IEEE Workshop on Applications of Computer Vision.

[11] Principal component analysis, http://en.wikipedia.org/wiki/Principal component analysis

[12] Jian , G. W., Eric, S. 2011. Active Learning for Solving the Incomplete Data Problem in Facial Age Classification by the Furthest Nearest-Neighbor Criterion. IEEE transactions on image processing.

[13] Hlaing, H.K. 2012. Subjective Age Prediction of Face Images Using PCA. International Journal of Information and Electronics Engineering, Vol. 2, No. 3,pp. 296-299

[14] Ahmed, N., Natarajan, T. 1974.Discrete Cosine Transform .IEEE Transactions on Computers, Vol.C-23.

[15] S. Bharkad and M. Kokare. 2011. Performance evaluation of distance metrics: application to fingerprint recognition. Int. J. of Pattern. Recogn. Artif. Intell (IJPRAI), Vol. 25, No.6, 2011, pp-777-806.

[16] S. Bharkad and M. Kokare. Modified FFT Features for Fingerprint Matching. Int. J. of Signal and Imaging Systems Engineering, Vol. 6, No. 3, pp. 150 - 157, 2013.

[17] S. Bharkad and M. Kokare. Hartley Transform based Fingerprint Matching. Int. J. of Information Processing, pp. 85-100. March-2012.

[18] S. Bharkad and M. Kokare. Rotated Wavelet Filtersbased Fingerprint Recognition. Int. J. of Patt. Recogn. Artif. Intell (IJPRAI),Vol. 26, No. 3, 2012.

[19] S. Bharkad and M. Kokare. Rotated Wavelet Filtersbased Fingerprint Recognition. Int. J. of Patt. Recogn. Artif. Intell (IJPRAI), Vol. 26, No. 3, 2012. 